\title{
5 Kaceytron and Transgressive Play on Twitch.tv
}

\section{Mia Consalvo}

"Fucking 4chan!" the woman on the Twitch stream exclaims in disgust. "Is that how you found my stream?!?! Were they posting fake nudes of me on 4chan?!" The Twitch stream shows an attractive young woman with glasses and a low-cut shirt on the left side of the frame; behind her and taking up the rest of the stream is her computer monitor's screen-showing at center a long and narrow, blurry but rapidly scrolling text chat window that features the term CUM DUMPSTER spammed over and over again. To the right of the text barrage, a browser window is opened to a page that is difficult to read but shows some Twitch interaction rules at the top and comments below. To the right of the streaming window (not pictured in figure 5.1), the overflowing chat channel crawls ever upward rapidly, making some comments difficult to read unless they are repeated (as many of them are) and in all caps. Someone has copy-pasted "KaceyPls THIS IS A KACEYTRON WAITING ROOM" multiple times into one comment that is nine lines long, and other chatters are rapidly posting "4chin," echoing (and mocking) Kaceytron as she shifts from pronouncing the infamous hate-filled site "4chan" into "4chin" in her rant. After 20 seconds, the scene jumps to Kaceytron announcing, "I'm one of the best streamers on Twitch.tv but sexists won't admit it because they're sexist." After a short rant, the stream cuts to Kaceytron playing a game; Kaceytron ranting about a news article displayed on a website; Kaceytron proclaiming about someone in the chat, "Look at this white fucking knight! Do you think that I'm gonna fuck you if you help me in Runescape?" After a few more seconds and images comes a static graphic informing the viewer that the live Kaceytron stream will be starting momentarilywhat we have just seen has amounted to a highlight reel of past Twitch moments chosen by Kaceytron for her viewers' amusement.

As part of a larger project investigating Twitch and live streaming, I first began watching Kaceytron's stream in early 2016 to understand more about women who stream gameplay on sites such as Twitch.tv. Twitch is a relatively new platform for individuals who want to live-stream themselves as they play videogames-showing their 


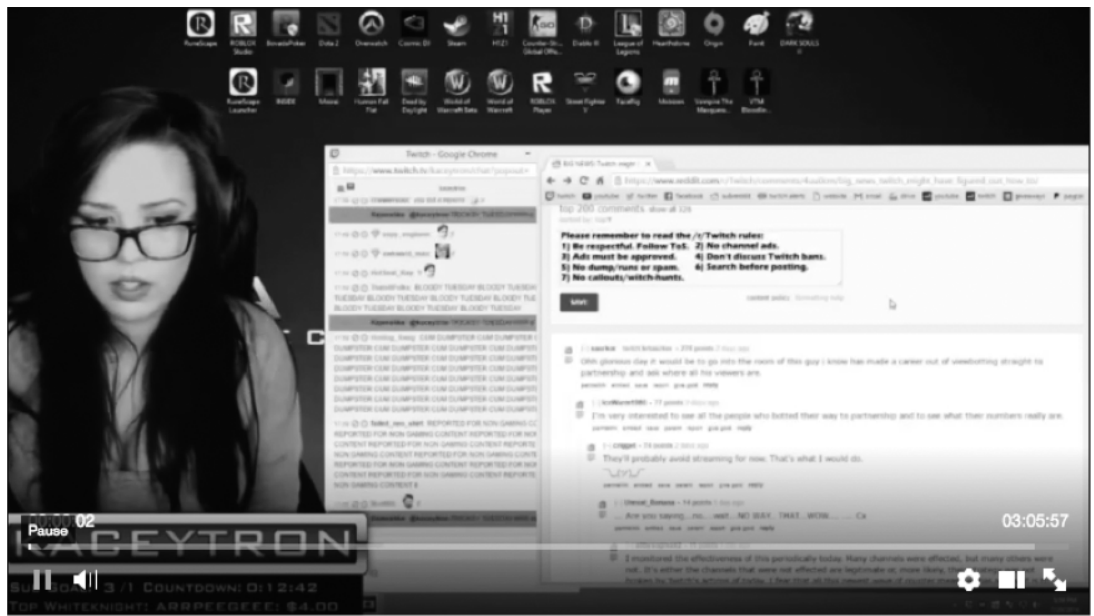

Figure 5.1

A recording of Kaceytron's Twitch stream from August 2016.

gameplay (via personal computer or console system) as well as audio and/or video of themselves as they play on a dedicated channel, where viewers can chat with them in real time. Twitch began as Justin.tv in 2007, a round-the-clock, life-streaming channel devoted to founder Justin Kan. The channel quickly expanded its service to multiple channels and allowed individuals to create accounts and stream for free-with the most popular gaming section branching off in 2011 and being rebranded as Twitch. tv. In 2014, the nongaming segments of Justin.tv ceased operations, and the creators sold Twitch to Amazon for $\$ 970$ million (Amadeo 2014). By the end of 2014, Twitch claimed 100 million unique monthly visitors, who were watching 1.5 million different broadcasters (Leslie 2015). The overwhelming majority of those Twitch streams focus on eSports titles such as League of Legends (Riot Games 2009) and Counter-Strike: Global Offensive (Hidden Path Entertainment 2012), and the predominant demographic attracted to Twitch is young, white, and male, primarily from North America (Leslie 2015; Quantcast 2016).

Despite the predominance of young men playing eSports titles, there has been an increasing number of other types of streamers (women streamers, queer streamers, streamers of color) as well as academic attention to those who are "variety streamers"-those who play a wide variety of videogames that are not necessarily competitive (Hamilton, Garretson, and Kerne 2014). Within that group, I became particularly interested in women streamers who received large amounts of online abuse on their streams. Yet I was hesitant to watch Kaceytron at first, for fear that her stream would 
confirm the worst stereotypes about Twitch and its viewers and would provide nothing new for our understanding of women, harassment, and online games. Adding to that hesitancy was the popular reaction to Kaceytron in the world of live streaming, where her reputation precedes her (MostlyBiscuit 2015). Kaceytron has been called many things, most of them not very polite. In terms of categorizing her, other streamers and YouTube viewers would probably refer to her as a "boobie streamer" or more charitably as a "girl streamer." It is difficult to find accurate information about her history, but according to her now defunct wiki site, she started streaming "World of Warcrafts" (as she calls her streams of playing World of Warcraft [Blizzard Entertainment 2004]) on Twitch in 2013, then switched to "League of Legions" (her term for her streams of playing League of Legends) later that year, and now works mainly as a variety streamer, meaning she plays many different games on her stream and hosts chat shows and news reports. Kaceytron lists her streaming schedule on her Twitch page: Wednesday through Sunday, starting at 5:00 p.m. CST and usually continuing for two to five hours. As of this writing, Kaceytron's streams have been viewed more than 29 million times; she has more than 472,000 followers (followers are free-anyone who wants to follow a streamer will be notified when she goes online), a YouTube channel with more than 67,000 subscribers, a subreddit with 1,487 readers, a Snapchat account, a Facebook page with more than 43,000 likes, and a Twitter feed with another 72,800 followers. Due to her success in attracting concurrent viewers, Kaceytron has achieved Partner status with Twitch, meaning followers can subscribe to her channel for $\$ 4.99$ per month (or more for premium subscriptions), which comes with such perks as being able to chat on "sub-only Sunday" streams, having access to subscriber-only emotes for chat, receiving a periodic newsletter from Kaceytron, and being eligible for periodic contests she puts together for subscribers. Likewise, viewers can send her one-time tips or donations while she is streaming, and she can also garner "cheers" from appreciative viewers-another of Twitch's revenue systems for streamers.

Two questions I asked in my project were, "Why would players put themselves in distress, and how does such content affect the playful attitude?" Based on my preliminary viewing of several different women streamers on Twitch as well as past views of eSports events and TwitchCon discussions about women and gaming, I thought I could investigate how and why women would voluntarily subject themselves to such online harassment, albeit to different degrees and in a variety of forms. When I first considered this question, I thought of comparing how Kaceytron manages the toxic atmosphere of Twitch (and of being a woman gamer online in general) and how other women streamers who do not seem to attract as much abuse (such as Annemunition and TheSpazzyProf) manage the responses to their streams. But in closely studying 
Kaceytron's streams in the context of her larger media presence-and especially her performance as Kaceytron-I concluded that her performance is complex enough that she deserves an entire chapter to herself. Kaceytron is an enigma-many viewers have asked in her stream and elsewhere if her performance is authentically her or if the onscreen presence we witness is simply a persona, a troll that is playing her audience. Digging around online provides only a few answers to these questions, but, ultimately, the Kaceytron performance is what matters-the simulation, if it is one, is the thing that counts. To investigate this performance, I regularly viewed Kaceytron's live streams during July and August 2016, taking detailed notes on five of those streams. I have also followed her Twitter stream and watched additional older videos she and others have posted about her on YouTube as well as on other sites online. I have read through her /r subreddit forum, searched the Internet for interviews and discussions about and with her, and examined her Facebook page. This has given me a deep view of the current incarnation of Kaceytron as well as some understanding of her past performance, but I cannot claim comprehensive knowledge of her entire streaming career because it started several years ago, and her practices have changed quite a bit from what it was before I began viewing and studying her performance. From this limited sampling, however, we can still ask how Kaceytron's performance is transgressive and specifically how it can be both toxic and playful at the same time. Following Chris Jenks, I define transgression as that which goes "beyond the bounds or limits set by a commandment or law or convention, to violate or infringe." Yet transgression is also "a deeply reflexive act of denial and affirmation" $(2003,2)$. It does double duty-at the same time that it violates a boundary (or convention), it affirms that same line. How do Kaceytron's transgressions operate? In this chapter, I discuss Kaceytron as a transgressive persona or performance and as a social media presence that is deeply tied to the contemporary gaming scene in the West and that implicates gender and gameplay in important ways.

\section{Women and Online Gaming: A Fraught History}

Although toxic gamer culture may seem a recent phenomenon to those who do not play games, it has deep and sustained roots and arguably goes back to the advent of digital games, when girls and women were marginalized as potential players in both arcades and in the home (Kocurek 2015). Writing in 2002 about the "gendered aspects of contemporary computer gaming," Jo Bryce and Jason Rutter point to how games in the home were not considered a girl's domain because "the technology and the gaming [were] controlled by the male player who assume[d] the role of expert by interpolating 
the female gamer into a subordinate role" $(2002,244,252)$. From there, it was a short step to discounting girls as players in public settings and then to actively denigrating them.

Illustrating this history, Fern Delamere and Susan Shaw write about Counter-Strike (Valve 2000), documenting the abuse that women faced while playing on public servers, explaining that, "unlike the male players, female gamers were frequent targets of abusive behaviours and discriminatory actions during game play" (2008, 290). Those actions included not just verbal abuse but also acts of sabotage against the female gamers' computers while at LAN events, such as hacking them to shut them down just before tournament play started and scrawling sexist messages on their computer screens (291). Yet the female participants Delamere and Shaw talked with were determined to persevere in their gameplay, often working to become even better players as well as setting up private servers where they could compete without experiencing abuse. It would be nice-but inaccurate-to say that this harassment has waned over time. Indeed, the activities surrounding the Gamergate controversy that began in 2014 simply shone a brighter, more public spotlight on what most gamers already knew-even as more girls and women were playing games, they were continually being insulted, trolled, harassed, threatened, bullied, and shamed for the simple act of playing (Consalvo 2012). In such a climate, the act of publicly playing a game as a girl or woman is not just a leisure activity but also a political act-a statement that gameplay is the

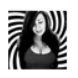

Duo w/ NickBunyun

kaceytron playing Counter-Strike: Global Offensive on Grand National Champions

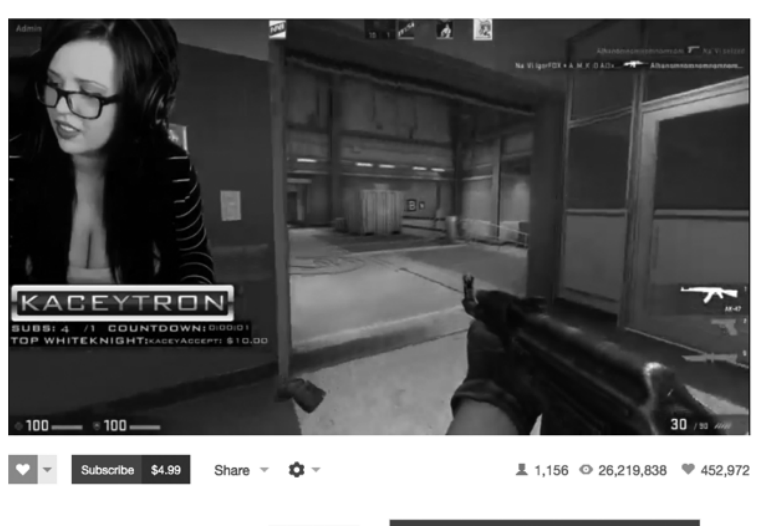

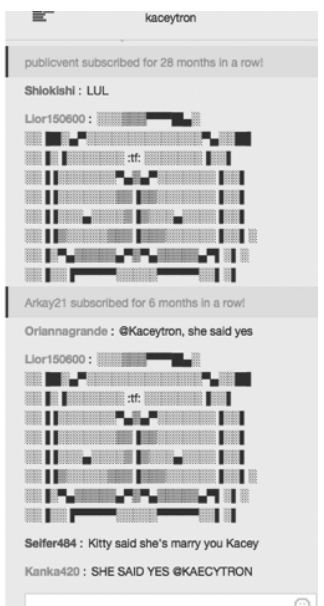

Figure 5.2

Kaceytron playing Counter-Strike: Global Offensive (Hidden Path Entertainment 2012) and the ASCII text spam sent to her by a viewer. 
domain of more than just boys and men. In that domain, I now focus on Kaceytron, who embodies and performs as "girl gamer" in multiple, contradictory ways.

\section{Watching Kaceytron}

Although past research has studied what happens to women players online and how those women respond, Twitch and its live-streaming capacity reconfigure the gameplay situation significantly, making it necessary to reconceptualize Kaceytron and her performance of play. Key to this reconceptualization is the recognition of a shift in focusfrom an act of play in a multiplayer (online or offline) situation to a display of single or multiplayer gameplay that is being performed primarily for an external audience. That performance is also mediated through the Twitch platform, which brings with it a unique set of limitations and affordances. Thus, Twitch itself becomes complicit in how Kaceytron performs (gendered) gameplay, in how her viewers respond to her and shape the stream, as well as in what type of community she and they are actively producing.

\section{Kaceytron Is Now Live ...}

Viewing a Kaceytron live stream is not for the faint of heart. A riot of manically scrolling text chat replete with semipornographic ASCII art and caps-locked insults moves on the right side of the screen; in the center of the streaming screen is gameplay, with animated and still graphics featuring sound periodically superimposed over the screen; and Kaceytron herself is positioned on the left. This layout is occasionally disrupted for the needs of a particular stream. During one stream in early July 2016, the screen was split into thirds, with Kaceytron in the left third, the game screen for Pokémon Go (Niantic 2016) in the middle third (played by Catcam, a regular stream participant and live streamer on his own channel), and the camera view for the game in the right third of the screen space. It was shortly after the launch of Pokémon Go in the United States, and Kaceytron was giving her audience access to a new game live, played "out in the wild" while she remained ensconced at her computer, actively commenting on the experience. Even though Kaceytron was not playing the game, she appeared resolutely in charge, directing Catcam-often bullying him-to do exactly as she wanted: "Look to the left! Look to the left! Look to the left! Now look forward. You should turn around. See? This is how people get hurt." Even though he complied with her orders, she continued to harangue him: "Don't get hit by a car-I'd be fucking banned from Twitch," she warned him as he wandered near a street. Shortly after that, she demanded, "Show me something impressive. Everybody already has that one [Pokémon]." While directing 
Catcam on his mission, Kaceytron was also reacting to the chatter that defines her stream. In response to a comment that she was lazy and having someone else do the work of catching Pokémon for her, she retorted, "I was catching Pokémon all fucking day, motherfucker." Later in the stream, as the chat got more abusive, she responded, "ok, chat's not fucking funny," and later told another chatter to "fuck off" in response to an insult. It is clear from watching even a short segment of a Kaceytron stream that Kaceytron meets viewer harassment with language in kind. In that way, Kaceytron performs "talking back" to her audience, refusing (mostly) to let them see that she is bothered by such abuse, even if it is directed at her in a perpetual stream. Although her chat "community" may be more voluminous in their ability to direct toxic language at her, Kaceytron retains the last word via her ability to verbalize her own retorts, giving her some authority over which voice is privileged (hers).

Layering on top of Kaceytron's own verbal commentary and the chat's textual assaults, the stream also features regular graphics (both static and gifs, all with accompanying sound effects) that appear over the screen's action in response to specific events. When someone renews their subscription, a graphic of Kaceytron in a hoodie with "Oh WOW" above it appears, accompanied by a sound file remarking "oh wow!" that employs Kaceytron's own voice. When the graphic appears, Kaceytron will announce that subscriber X has subscribed for " $x$ years in a row! Thank you!" Interspersed with news of subscription renewals, which can become formulaic, are other graphics that announce donations. Any viewer (whether subscriber or viewer) can donate any amount of money to Kaceytron and include a message with it that will also appear on her stream's screen. She reads these out, too, usually verbatim. The vast majority of these donations are ways to further insult Kaceytron, such as "Kacey I'm so close please give me permission to cum. Thanks, Daddy." Very occasionally-as in this caseKaceytron refuses to read them aloud and will simply respond to the donor, "Go fuck yourself," even as the donation and text appear on screen for all to see. This is one way for audience members to gain a more prominent "voice" in her stream, vaulting their commentary out of the rapidly scrolling chat window and overlaying her gameplay, briefly placing their speech on a level equal to Kaceytron's. There's even the promise that Kaceytron will read aloud the missive (provided it is not too offensive even for Kaceytron), granting it further legitimacy. Yet such speech comes at a literal priceaudience members must pay a donation to have their words appear in this prime position. Whereas Kaceytron can chatter on unheeded, her audience members must pay if they want to be heard, even if it is to insult or mock what is going on.

Last-but definitely not least-below Kaceytron is a counter of new subscribers as well as a space to list the "TOP WHITEKNIGHT" of the stream. This space is reserved 


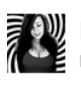

Duo w/ NickBunyun

kaceytron playing Counter-Strike: Global Offensive on Grand National Champions

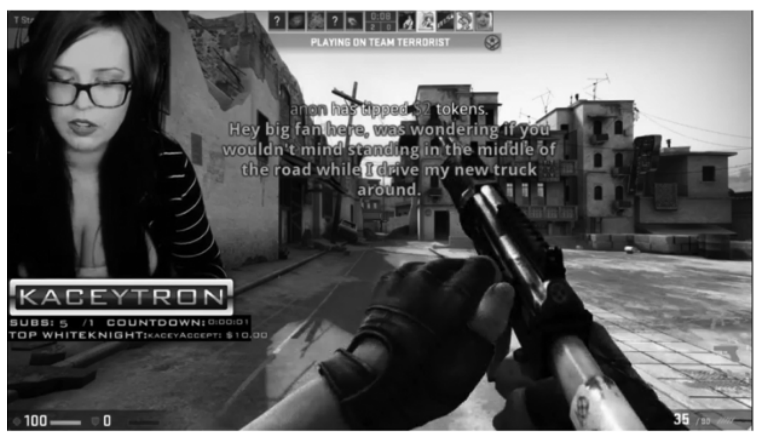

C. Subscribe sa.99 Share a
$1.203 \circ 26.219 .838 * 452.972$

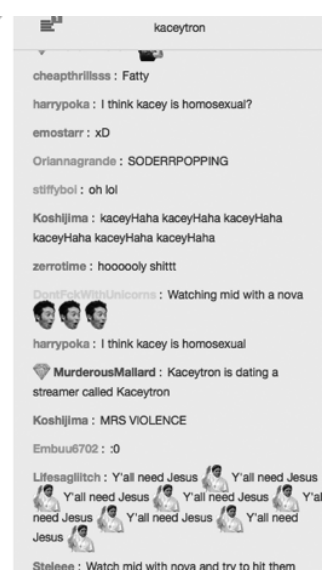

Figure 5.3

Donation texts appear on screen.

for a chatter (or donor) who appears to defend Kaceytron excessively from those who are harassing her or for someone who is too grandiose in their praise of Kaceytron. When that happens, Kaceytron calls the offender out, directing the chat: "Can I get a no WhiteKnight in the chat?" Upon hearing this, subscribers, who have access to special chat icons, will spam an icon of a "banned" white knight chess piece that they have access to for just such occasions. The banned white knight is a particularly interesting feature because it is a material instance of Kaceytron's dissatisfaction with any rhetoric that she must be protected or defended by men-that she is unable to take care of herself or believe in her own ability to play. Echoing findings from Jenny Sundén and Malin Sveningsson (2012) about female World of Warcraft players, Kaceytron asserts her own agency as a player and a streamer, refusing even the more benevolent forms of sexism in games, such as the belief that women in such spaces are in need of male protection.

Yet Kaceytron also either welcomes or allows quite a bit of disturbing content in her stream. Based on my notes on past streams and recordings of other streams, it is easy to see why Twitch has been labeled as toxic soup for female streamers. The chat and donation comments that appear in one 3-hour stream are a barrage of offense-mixing copy-pasted ASCII art of boobs and dicks with repetitions of insults in all-caps, the occasional $<$ message deleted $>$ for a message that was too foul even for Twitch's permissive chat filters, and the random other slights and comments that make up her stream. Yet other than telling the particularly obnoxious ones to "fuck off," Kaceytron does little to staunch the flow. Raunch and abuse are instead allowed, normalized, and then capitalized on as they become signature elements of the Kaceytron brand. 
We can see this logic at work in how Kaceytron sets up the rules for engagement in her space. Many streamers have a list of "chat rules" for their channel, which typically include types of speech or behavior that they do not allow. For example, Annemunition's site (with more than 175,000 followers) lists her chat rules as "Don't spam, fight with others, or use hate speech. Be courteous, respectful, and play nice with the other viewers." Likewise, TheSpazzyProf (with about 20,000 followers) has an extensive list of "rules" that include "Don't be rude to me, my mods or each other" "English only!!" "Profanity is allowed, but hate speak is not. No racist, homophobic, transphobic, misogynistic, sexually graphic, or otherwise ignorant speech," and "If someone is trolling, do not engage them or acknowledge their existence. Allow the streamer or mods to take care of business. Just sit back and watch the ban-hammer swing!" In contrast, Kaceytron lists only three rules at the time of this study: "1. Freedom of Speech for everyone! 2. Do not advertise other streams. 3. Do not link pornographic images." In Kaceytron's streams, trolls are allowed to speak, and the abuse runs wild-on both sides. On another night in July 2016, Kaceytron was setting up a game of CounterStrike: Global Offensive with a well-known player, and the chat was typical for one of her streams. Comments included "she has an ugly face," "CAN I SEE YOUR BOOBS" repeated over and over, "show tits bitch," "where's the ugly cunt," and "FUCKING READ MY COMMENT YOU CUM DUMPSTER." In this situation, Kaceytron directed blame to the visiting player-suggesting that it was his viewers who were clogging up her chat with out-of-control remarks-rather than acknowledge the toxicity of her own stream. But even in that case she did not appear offended by the noxious material—she was simply intent on pointing out "who" was to blame for it.

Toxicity has become the element that defines Kaceytron's stream, far more than her low-cut shirts or her inability to play very well. Or, rather, her cleavage and play style are props that prompt the toxicity that ensues. Other researchers have investigated how online harassers have monetized hate via systems that encourage viewers to donate money to see more abuse and harassment (Mortensen 2016). Kaceytron's stream is not of the same order-she does not attack viewers or others who offer no provocation; rather, she has built a system where viewers pay her to direct abuse toward her via multiple formats. They can subscribe monthly; they can give one-time donations; or they can cheer her (although this system is not really useful for insults or harassment). Likewise, other viewers can watch the ensuing toxicity-for free-as well as participate in it, adding to and becoming the "free to play" toxic content that provides further enjoyment for those who do pay and that helps to continue defining the Kaceytron brand as a toxic "shit show." 


\section{Gaming Capital, Gender, and Kaceytron}

One of the ways Kaceytron actively transgresses is in her performance as a "girl gamer." A perpetual issue for girls and women who play is that they are not recognized as legitimate players or gamers-they are thought of as faking interest for attention or money or both, or they are dismissed as being bad at actually playing the games (Sundén and Sveningsson 2012). Over and above how Kaceytron interacts with her viewers generally, her actual gameplay complicates her persona. During many streams that I viewed of Kaceytron during the summer of 2016, she played badly, dying repeatedly in Counter-Strike; skipping important cutscenes in This Is the Police (Weappy Studio 2016), which made later sections difficult to play; and reading the introductory text in another game so slowly that it disappeared and she had to relaunch the game to finish reading it.

Kaceytron's actions undermine her continual proclamations that she is an excellent player. She will regularly tell viewers that she is a pro and that she excels in multiple types of gameplay. Yet coupled with her proclamations of talent are statements that support her seemingly poor gameplay. Those statements are often willful dismissals of certain forms of gaming knowledge-gaming capital—and especially common knowledge that anyone tuning in to her stream should easily know and recognize (Consalvo 2007). So, for example, she refers to the "meta" of the game League of Legends as "the meet-a," and she also talks about "beta" versions of games as "beet-a" versions, willfully mispronouncing each term. Such actions would seem to present a surface reading of Kaceytron as a bad player with delusions of grandiosity.

Yet when Kaceytron did play League of Legends early in her streaming career, she participated in ranked play over multiple seasons. To participate in ranked play, players have to attain level 30 in the game (which can take a significant period of time) and must own a set number of champions-the avatars that the game employs. Kaceytron's website includes many guides to playing different champions, with videos that show her achieving 26 kills (an impressive amount) with one champion in particularJinx-yet advice such as "IF YOU ARE LOOKING FOR A CHAMPION THAT HAS CUTE SKINS THIS IS NOT THE CHAMPION FOR YOU" and "I HAVE RECEIVED A LOT OF CRITICISM FOR NOT USING THE 'PROPER' ABILITY NAMES. I DO THIS INTENTIONALLY, AND I THINK THAT IT IS A GREAT PRACTICE FOR LEARNING A CHAMPIONS [sic] ABILITIES BY RELATING IT TO SOMETHING YOU ARE MORE FAMILIAR WITH." Although her recent ranking has been lower, she also has videos showing that she attained Gold V status, which is in the top 10 percent of ranked League of Legends players. Kaceytron therefore poses a dilemma for the persona of the girl gamer-Is she good 
at games or bad at games, and does it matter? Many of the more recent games that she plays are considered significantly less challenging than League of Legends, yet she still performs badly in all of them. Her rhetoric proclaims her superior ability, which is (most often) belied by actual displays of her gameplay efforts, often featuring failure. Kaceytron refuses to consent to the pigeonholing of her as a "bad" girl gamer, though, but that refusal then further feeds the toxicity of her stream: viewers regularly tell her to delete her account, to stop playing games, and so on. Her seemingly off-the-cuff references to the context surrounding games shows knowledge of that complexity to a degree-she is aware of the meta, aware of class strategies to follow when playing World of Warcraft. But her performance of gaming-capital knowledge appears fragmented or just plain wrong. If she knows these things, why does she not get them right? Rather, a more appropriate description would be that she screws up, she fails, but she is still successful in performing play-merely a different form of play: playing with her viewer's expectations.

\section{Kaceytron, Griefing, and the Twitch Community}

Another way of understanding Kaceytron's transgressive play practices would be to see them as a form of griefing - albeit of a different sort than is written about in traditional studies of player culture (Foo and Koivisto 2004; Lin and Sun 2005). According to Chek Yang Foo and Elina Koivisto, the goal of griefing in playing a videogame is to antagonize other players or destroy their progress or both rather than to get ahead or advance one's position in the game. In studying massively multiplayer online role-playing game players and particular activities that may or may not be considered griefing by the community, they conclude that for players "an activity is clearly griefing if the actor has little direct gains from the act, or if the act is repeated, or if another griefing type accompanies the act" $(2004,250)$. Holin Lin and Chuen-Tsai Sun investigate "whiteeyed" players in Taiwan, who act in ways similar to griefers. They concur that "grief players are generally considered the deviants in gaming societies; they break the laws (codes and rules of conduct) of their game worlds, violate the norms and etiquettes of their communities" $(2005,2)$. Yet in their investigation of problematic play activities, Lin and Sun discovered that there is a difference between who the imagined griefers are and who actually indulges in grief play. They point out that "the only way to identify a griefer is when he or she comes out" and that the labeling of griefers is ultimately about naming those "outside" the game so that "[adult] players can redefine gaming in their own way" $(2005,11)$. 
Kaceytron both fits and does not fit descriptions of griefers. Importantly, past theorizations have defined griefers as players griefing other players who are playing within the same game. Many of Kaceytron's recent streams have featured single-player games, where no griefing can really happen. Past videos that show her playing League of Legends demonstrate that her in-game gameplay may or may not have been optimal, depending on the match, but she did not go out of her way to make other players suffer-she performed "playing the game" with the intent to win, although whether she was really playing poorly on purpose is unknown. Unintentional griefing as described by Lin and Sun was a possibility in this case-if we acknowledge that Kaceytron's performance as a poor player who thinks she is excellent (by choosing suboptimal strategies and actions) was designed to enrage her teammates during a competitive event. But as Lin and Sun point out, we cannot know someone is really griefing unless the player self-announces their role as such, and Kaceytron resolutely refuses to do so both during and outside of her play sessions.

More importantly and interestingly, Kaceytron's potential griefer status arises from how she communicates with and performs for her viewers, many of whom behave like trolls and whom she often "trolls back." For example, in a stream on July 15, 2016, Kaceytron joined up with Nick Bunyun, another popular streamer and Counter-Strike player, to play the game. Although the majority of her stream time was not really focused on the gameplay-even when she and Nick were actively playing — it is useful to highlight a few moments to see how Kaceytron operates. When they were finally starting the game, after almost an hour of setup and chat, Kaceytron announced, "I'm really good at the hostage maps." This was still during the warm-up phase, but her statement put Nick and the stream on notice that she (presumably) both knows something about the game (what kinds of maps are available) and has preferences and expertise relative to them. However, shortly after a match began, Kacey's avatar was shot and she died. She commented, "Not sure where that came from." She respawned and then quickly died again, announcing, "Okay we need to turn this game around," as if the rest of the team were at fault. After dying yet another time, she responded, "I think that person is using aimbots" - a type of cheat that lets players automatically aim their guns.

The chat in reaction to Kaceytron's performance on this occasion was predictable for her stream. Numerous chatters told her that she was a terrible player, that she sucked, that she should uninstall the game and kill herself, and that "they're good, you're not." Yet Kaceytron was undeterred, telling Nick that his viewers were going into her chat to abuse her, that they did not know her, and that "you need to get your viewers in line." She concluded all this was happening because "they don't like to see girls who 
are better than them." At this point, it was difficult to know if Kaceytron was indeed a poor player with delusions of grandeur or possibly someone with a run of bad luck at the start of the game. Even her guest partner, Nick, at one point said, "You're going the wrong way," to which she replied, "I'm taking a short cut." After a period of time, she either started to improve or caught a break and was dying less often. At that point, the focus of the stream shifted back away from the game itself and onto the conversation between Kaceytron and Nick, both of whom seemed intent on asking each other absurd questions and replying in as obtuse a way possible.

In this segment, a few things are going on. Almost as a given are streams of borderline (and not so borderline) obscene insults continuously scrawling up the chat screen; such insults were omnipresent during this exchange. But rather than acknowledge as legitimate the grief and trolling sent her way, Kaceytron instead hurls it back, denying its relevance and refusing to acknowledge that she herself is a griefer. She is not a poor player, and her viewers are not the problem-she is only there to play and have a good time. The abuse is therefore-according to Kaceytron-coming from someone else's fans, who are "obviously" hostile to a woman who is better than they at a particular game. This statement can be hard to deny-research has found that women gamers do in fact face abuse, particularly when they beat male gamers in competitive games (Delamere and Shaw 2008). Kaceytron, rather than flee from abuse, discredits its source. She further refuses the terms of engagement-that she is a poor player. She instead confidently asserts that she is a superior player. However, the audience knows-or thinks they know-that she is not as good as she believes. Is she really bad and thus lying to herself? Is she a good player but deliberately messing up? Is she trolling the trolls? It is impossible to tell, which makes the performance so multilayered and tantalizing. Who is winking at whom?

\section{Trolling Twitch's Streamer Culture}

There is a constant back and forth with Kaceytron, some of her actions signaling she knows nothing, yet others suggesting she might just be playing around. It would be easy at this point to dismiss Kaceytron as someone who does indeed make money by being a girl in a low-cut shirt who is (possibly) decent at games but vastly inflates her skill through her rhetorical performances and flourishes. But alongside Kaceytron's mispronunciations and mistakes are savvy and incisive critiques of the very performance she is enacting. She regularly calls out disingenuous activities that occur in the Twitch live-streaming community_in her gaming streams, in "news updates," on Twitter, and elsewhere. In early August 2016, she tweeted about how "being a girl is 
so hard," a tweet that featured what her critics would suggest is her normal persona at work.

Such tweets easily support the "Kaceytron as troll" and "Kaceytron as boob streamer" narratives. Yet I believe Kaceytron also engages in what Kishonna Gray calls "resistance griefing," defined as using the resources available to oneself to derail "negative experiences and fight back against the dominant structures within the [game] space" (2013). So, for example, Kaceytron also ranted on Twitter a few days after the stream on July 15 about Twitch streamers who engage in ethically (and legally) questionable practices (as shown in figure 5.5).

9. kaceytron @kaceytron · Aug 6

Being a girl is so hard $u$ have to do hair and makeup so expensive pls like if $u$ agree that being a girl is hard \& girls need to be respected

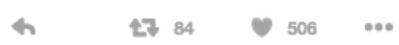

Figure 5.4

Tweet from August 6, 2016.

9. kaceytron @kaceytron - Jul 16

or when I did a charity drive while simulatenously losing hundreds of

dollars at black jack, oh wait never.... but I have tits

h $\quad 2725 \quad 0212 \quad \ldots$

9. kaceytron @kaceytron · Jul 16

trying to remember when I asked my

viewers for money to buy a car, or go to a

con, or manipulated by viewers into a donation war

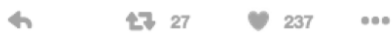

8. kaceytron @kaceytron - Jul 16

The double standard in this community is fucking disgusting. I'm a scam artist for having tits, not sponsored by g2a, no subtrain, no tts

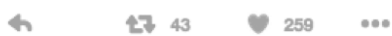

Figure 5.5

Kaceytron reacts to Twitch streamers' practices. 
Kaceytron's rant about "scam artists" on Twitch exposes the double standard she is accused of - that by "showing off her tits," she is the supposed scam artist rather than those who engage in practices that are much more directly about getting money out of one's viewers, such as actively soliciting donations on screen, promising perks for cash, and so on. During her streams as well as on Twitter, viewers and followers regularly excoriate her for a lack of skill and for "taking views away" from more "legitimate" streamers - both female and male - a claim made against many female streamers who are conventionally attractive (Hernandez 2016). In addition to taking views "away" from other streamers, "boobie streamers" can also be blamed for normalizing the toxic attitude toward women streamers on Twitch. Perhaps the best example of this argument can be found in the YouTube video Dear Female Streamers made by popular Twitch streamer Sky Williams. The video, which has more than 1.3 million views as of this writing, is a four-plus minute long rant by Sky (a black gay man) about how female streamers who show their boobs are now the "standard" by which all female streamers are judged and how the bad behavior they engender in male viewers is transferred to all female streamers as a result (S. Williams 2015). In a later Twitch stream, however, Sky clarified that Kaceytron is not one of the streamers he is targeting because "she is doing it for satire. Obviously" (Generation Blue 2015).

What is also key to point out and perhaps lends credence to the idea that Kaceytron is a resistance griefer who knows exactly what she is doing is that even as she mispronounces key gaming terms that any "core" gamer should know, she is at the same time adept at using the lingo of monetized Twitch streamers in her tweets. The acronym TTS stands for the "text-to-speech" function that computer users can engage to have typed text read aloud. In the Twitch community, TTS has become an entertaining promotional tool when it is turned on by some streamers to read out donation messages coming in from viewers. The results can be both funny as well as obscene, but Kaceytron points out that they also serve as an easy way to gain donations for the streamer in charge. She likewise points to the use of "subtrains," which occur when viewers of a particular stream start subscribing every five minutes and a count is kept to see how high the "subtrain" can go. Finally, Kaceytron mentions the g2a website, which offers to sponsor certain Twitch streamers to promote the site, where players can buy and sell game keys that have questionable origins. (She also posts an email allegedly showing g2a asking her to become a sponsor of the site, which she refused.) Kaceytron's succinct use of all these terms in one tweet demonstrates that she does possess gaming capital of a key sort-the capital related to the world of professional Twitch streamers. 


\section{Conclusions}

Returning to the question of how Kaceytron's stream and performance can be considered transgressive, we can see how she violates multiple boundaries-what "girl gamers" should look and act like, how a stream's chat should be managed, and what a "successful" stream should be. In one of the few interviews with Kaceytron that I could find, she explains that although part of what she is doing online is based on her own personality and interests, she views her stream mostly as "long-form improv" (MostlyBiscuit 2015). Such a categorization makes sense-the persona she has created is so over the top that it begs not to be taken seriously, even as it contains dashes of truth and incisive critique. Viewers seem to recognize this-constantly talking online about how she is a troll and not to fall for her act-even as they end up viewing and falling into the trap of hectoring her for that very performance. The Kaceytron that we watch-looking young and attractive, showing cleavage, burping, swearing, and playing videogames well or poorly-is a perpetual work in progress that challenges our ideas about what women streamers should be. She says being a girl gamer is hard, but she is awesome at it, even as she dies multiple times. Her spectators subscribe for months on end and offer unending streams of donations simply to share her screen space and talk back to her in some way. She refuses to be silenced by abuse or even to hide the abuse-embodying a form of the resistance griefer that Gray (2013) has written about. And when the act becomes too consistently one-sided, ultimately reifying the normative boundaries for proper streaming behavior, Kaceytron slides into an incisive critique of the Twitch community, calling to task those who employ viewbots or promote gambling sites or other practices that are either ethically or legally questionable. Her words and her actions transgress in multiple ways, even as they are cloaked in the everydayness of a girl gamer in a low-cut shirt who is not afraid to swear back at those who insult her. 